KUNS- 1611

\title{
Ferromagnetism of quark liquid
}

\author{
Toshitaka Tatsumi \\ Department of Physics, Kyoto University \\ Kyoto 606-8502, Japan
}

\begin{abstract}
Usually it is believed that the Hartree-Fock state of quark matter is a Fermi gas state with no polarisation of spins. We examine the possibility of the polarised quark liquid interacting with the one-gluon-exchange interaction. It is suggested that the Hartree-Fock state shows a spontaneous magnetic instability at low densities through the same mechanism as the appearance of ferromagnetism in electron gas. Metastability of the polarised quark liquid is also discussed.
\end{abstract}

PACS: 04.40.Dg, 07.55.Db, 12.38.Bx, 26.60.+c, 97.60.Jd

Keywords: neutron stars, ferromagnetism, quark matter, magnetic field 
The physical origin of the strong magnetic field in neutron stars is a long-standing problem [1]. Recent discovery of "magnetars" [2,3] - neutron stars with magnetic field strengths in excess of $B_{c r}=m_{e}^{2} c^{3} / e \hbar \sim 4.4 \times 10^{13} \mathrm{G}$ and typically $\sim 10^{15} \mathrm{G}$ - also stimulate our interest in the origin of their strong magnetic field. It has been widely believed that the magnetic field of neutron stars is inherited from the progenitor stars [1]. However, the extraordinary magnetic field in magnetars seems to enforce our reconsideration of its origin. Inside neutron stars, hadronic matter exists beyond the nuclear density $\left(n_{0} \simeq 0.16 \mathrm{fm}^{-3}\right)$, so that it may be interesting to consider the hadronic origin of the magnetic field.

In this Letter we examine the possibility of the spin-polarised quark liquid by the use of the Fermi liquid theory, where quarks interact on each other by the one-gluon-exchange (OGE) interaction. Since the system should be totally color singlet (neutral), there is left only the exchange energy within the Hartree-Fock approximation. It has been suggested that three-flavor quark matter (strange quark matter) can be stable or metastable for a reasonable range of QCD parameters [4]. The appearance of strange $(s)$ quarks is favored at and above the nuclear density due to the reduction of the kinetic energy of the system and the specific feature of OGE interaction; the exchange energy is negative for massive strange quarks at low densities, while it is always positive for massless $u$ and $d$ quarks. This feature is very similar to the Coulomb interaction for electron gas. In the following we consider the possibility of ferromagnetism in strange quark matter on the analogy with electron gas.

In 1929 Bloch first pointed out the possibility of ferromagnetism of electron gas at low density [8]: electrons interact on each other by the exchange Coulomb force, which contribution is attractive and becomes dominant over the kinetic energy at low density due to their different density dependence. Then the unpolarised electron gas suddenly turns into the completely polarised state at the critical density (the first order phase transition). The critical density is determined by the mass of electrons and the QED coupling constant $\alpha=e^{2} / 4 \pi$; lower density, where the nonrelativistic approximation works very well, is favored for the spontaneous magnetisation. This idea has been confirmed theoretically by the use of the Green function Monte-Calro method [9]. 
Therefore if the exchange energy is attractive and dominant over the kinetic energy at some density, there should appear ferromagnetism also in strange quark matter, as in electron gas. The idea can be put into a relativistic formulation utilizing the polarisation density matrix [10]. Then we shall see a difference between theories of electron gas and quark liquid. The scattering amplitude of two quarks becomes momentum dependent, and includes not only the spin-nonflip term but also the spin-flip one due to the lower component of the Dirac spinor, the latter of which vanishes in the non-relativistic limit. Also, OGE interaction itself include the spin-dependent (magnetic) interaction as well, which becomes effective in the relativistic regime, besides the Coulomb-like (electric) interaction. These features somewhat change the mechanism of polarisation in quark liquid from in electron gas. Actually we shall see that there is a possibility of spontaneous magnetisation even in the relativistic regime, where the exchange energy contribution is no longer attractive, by a different reason from electron gas.

Consider the spin-polarised quark liquid with the total number density of quarks $n_{q}$ ( we denote the number densities of quarks with spin up and down by $n_{+}$and $n_{-}$, respectively f , and introduce the polarisation parameter $p$ by the equations,

$$
n_{+}=\frac{1}{2} n_{q}(1+p), \quad n_{-}=\frac{1}{2} n_{q}(1-p),
$$

under the condition $0 \leq p \leq 1$. We assume as usual that three color states are occupied to be neutral for each momentum and spin state. The Fermi momentum in the spin-polarised quark matter then is

$$
k_{F}^{+}=k_{F}(1+p)^{1 / 3}, \quad k_{F}^{-}=k_{F}(1-p)^{1 / 3}
$$

with the Fermi momentum for the unpolarised matter, $k_{F}=\left(\pi^{2} n_{q}\right)^{1 / 3}$. The kinetic energy density is given by the standard formula,

\footnotetext{
${ }^{1}$ We,hereafter, consider one flavor quark matter, since OGE interaction never mix flavors.

${ }^{2}$ The meaning of spin up and down should be carefully defined in the relativistic theories. Here we call them in the rest frame of each particle. Their precise meaning is given later.
} 


$$
\epsilon_{k i n}=\frac{3}{16 \pi^{2}} \sum_{i= \pm}\left[k_{F}^{i} E_{F}^{i}\left(2 k_{F}^{i 2}+m_{q}^{2}\right)-m_{q}^{4} \ln \left(\frac{E_{F}^{i}+k_{F}^{i}}{m_{q}}\right)\right] \text {, }
$$

with the Fermi energy $E_{F}^{i}=\left(m_{q}^{2}+k_{F}^{i 2}\right)^{1 / 2}$.

The interaction energy is given by the OGE interaction. There is no direct energy contribution (Hartree term) and left only the exchange energy contribution (Fock term). To characterize the degeneracy of the plane wave solution $u^{(\alpha)}(k)(\alpha=1,2)$ for a positive energy state, we introduce a space-like 4-pseudovector $a^{\mu}$ which in the rest frame is twice the three-dimensional mean spin vector $\boldsymbol{\zeta}$ with $|\boldsymbol{\zeta}|=1, a^{\mu}=(0, \boldsymbol{\zeta})$. The 4-vector in any frame, where the particle is moving with the momentum $\mathbf{k}$, is found by a Lorentz transformation from the rest frame, and

$$
\mathbf{a}=\boldsymbol{\zeta}+\frac{\mathbf{k}(\boldsymbol{\zeta} \cdot \mathbf{k})}{m_{q}\left(E_{k}+m_{q}\right)}, a^{0}=\frac{\mathbf{k} \cdot \boldsymbol{\zeta}}{m_{q}}
$$

with $E_{k}=\left(m_{q}^{2}+|\mathbf{k}|^{2}\right)^{1 / 2}$. By choosing $\boldsymbol{\zeta}$ along the $z$ axis, $\boldsymbol{\zeta} \equiv(0,0, \pm 1)$, we see that the spinors $u^{(\alpha)}(k)$ are eigenstates of the operator $-W \cdot a / m_{q}$, where the 4 -vector $W^{\mu}$ is the Pauli-Lubanski vector, $W^{\mu}=-\frac{1}{4} \epsilon_{\mu \nu \rho \sigma} k^{\nu} \sigma^{\rho \sigma}$. For the standard representation of $u^{(\alpha)}(k)$ [11], the eigenvalue is $+1 / 2(\operatorname{spin}-u p)$ for $u^{(1)}(k)$, and $-1 / 2($ spin-down $)$ for $u^{(2)}(k)$. Then the polarisation density matrix $\rho$ is given by the expression,

$$
\rho(k, \zeta)=\frac{1}{2 m_{q}}\left(\not k+m_{q}\right) P(a), \quad P(a)=\frac{1}{2}\left(1+\gamma_{5} \not h\right),
$$

which is normalized by the condition, $\operatorname{tr} \rho(k, \zeta)=1$.

Let us consider the interaction between two quarks with momenta, $k$ and $q$, and the different spin vectors, $\boldsymbol{\zeta}$ and $\boldsymbol{\zeta}^{\prime}$, respectively. They are specified by the polarisation density matrices, $\rho(k, \zeta)$ and $\rho\left(q, \zeta^{\prime}\right)$. The Landau Fermi-liquid interaction $f_{\mathbf{k} \zeta, \mathbf{q} \zeta^{\prime}}$ is related to the two-particle forward scattering amplitude [12],

$$
f_{\mathbf{k} \zeta, \mathbf{q} \zeta^{\prime}}=\frac{m_{q}}{E_{k}} \frac{m_{q}}{E_{q}} \mathcal{M}_{\mathbf{k} \zeta, \mathbf{q} \zeta^{\prime}}
$$

where $\mathcal{M}_{\mathbf{k} \zeta, \mathbf{q} \zeta^{\prime}}$ is the usual Lorentz invariant matrix element. The color symmetric matrix element is given by the exchange term only; the direct term vanishes because the color symmetric combinations $\left(\sim \operatorname{tr} \lambda_{a}\right)$ does not couple to gluons. Thus 


$$
\begin{aligned}
\mathcal{M}_{\mathbf{k} \zeta, \mathbf{q} \zeta^{\prime}}^{s} & =-g^{2} \frac{1}{9} \operatorname{tr}\left(\lambda_{a} / 2 \lambda_{a} / 2\right) \bar{u}^{\left(\zeta^{\prime}\right)}(\mathbf{q}) \gamma_{\mu} u^{(\zeta)}(\mathbf{k}) \bar{u}^{(\zeta)}(\mathbf{k}) \gamma^{\mu} u^{\left(\zeta^{\prime}\right)}(\mathbf{q}) \frac{-1}{(k-q)^{2}} \\
& =\frac{4}{9} g^{2} \frac{1}{4} \operatorname{tr}\left[\gamma_{\mu} \rho(k, \zeta) \gamma^{\mu} \rho\left(q, \zeta^{\prime}\right)\right] \frac{1}{(k-q)^{2}} .
\end{aligned}
$$

Substituting Eqs. (幽) and (5) into Eq. (7), we finally find

$$
\begin{aligned}
\mathcal{M}_{\mathbf{k} \zeta, \mathbf{q} \zeta^{\prime}}^{s} & =\frac{2}{9} g^{2}\left[2 m_{q}^{2}-k \cdot q-(\mathbf{k} \cdot \boldsymbol{\zeta})\left(\mathbf{q} \cdot \boldsymbol{\zeta}^{\prime}\right)+m_{q}^{2} \boldsymbol{\zeta} \cdot \boldsymbol{\zeta}^{\prime}\right. \\
& +\frac{1}{\left(E_{k}+m_{q}\right)\left(E_{q}+m_{q}\right)}\left\{m_{q}\left(E_{k}+m_{q}\right)(\boldsymbol{\zeta} \cdot \mathbf{q})\left(\boldsymbol{\zeta}^{\prime} \cdot \mathbf{q}\right)+m_{q}\left(E_{q}+m_{q}\right)\left(\boldsymbol{\zeta}^{\prime} \cdot \mathbf{k}\right)(\boldsymbol{\zeta} \cdot \mathbf{k})\right. \\
& \left.\left.+(\mathbf{k} \cdot \mathbf{q})(\boldsymbol{\zeta} \cdot \mathbf{k})\left(\boldsymbol{\zeta}^{\prime} \cdot \mathbf{q}\right)\right\}\right] \frac{1}{(k-q)^{2}}
\end{aligned}
$$

after some manipulation of $\gamma$-matrix algebra. If we choose both $\boldsymbol{\zeta}$ and $\boldsymbol{\zeta}^{\prime}$ along the $z$ axis in parallel, $\boldsymbol{\zeta}=\boldsymbol{\zeta}^{\prime}=(0,0, \pm 1)$, we have the spin-nonflip amplitude $\mathcal{M}_{\mathrm{kq}}^{\text {s,nonflip }}$, while if we choose them in anti-parallel, $\boldsymbol{\zeta}=-\boldsymbol{\zeta}^{\prime}=(0,0, \pm 1)$, we have the spin-flip amplitude $\mathcal{M}_{\mathrm{kq}}^{\text {s,flip }}$. Each form of the spin-nonflip or spin-flip amplitude is complicated, but the average of them gives a simple form,

$$
\overline{\mathcal{M}}_{\mathbf{k q}}^{s}=\frac{2}{9} g^{2} \frac{2 m_{q}^{2}-k \cdot q}{(k-q)^{2}}
$$

which is nothing but the matrix element for the unpolarised case [12]. In the nonrelativistic limit, $m_{q} \gg|\mathbf{k}|,|\mathbf{q}|$, the matrix element is reduced into the form,

$$
\mathcal{M}_{\mathbf{k} \zeta, \mathbf{q} \zeta^{\prime}}^{s}=\frac{2}{9} g^{2} \frac{m_{q}^{2}\left(1+\boldsymbol{\zeta} \cdot \boldsymbol{\zeta}^{\prime}\right)}{(k-q)^{2}}
$$

so that there is no correlation between quarks with different spins. On the other hand, there is some correlation included even in the Hartree-Fock approximation for the relativistic case.

After summing up over the color degree of freedom and performing the integrals of the Fermi-liquid interactions, $f_{\mathbf{k q}}^{s, \text { nonflip }}=\left(m_{q} / E_{k}\right)\left(m_{q} / E_{q}\right) \mathcal{M}_{\mathbf{k q}}^{s, \text { nonflip }}$ and $f_{\mathbf{k q}}^{s, f l i p}=$ $\left(m_{q} / E_{k}\right)\left(m_{q} / E_{q}\right) \mathcal{M}_{\mathbf{k q}}^{s, f l i p}$, over the Fermi seas, we have the exchange energy density $\epsilon_{e x}$ consisting of two contributions,

$$
\epsilon_{e x}=\epsilon_{e x}^{\text {nonflip }}+\epsilon_{e x}^{f l i p}
$$

The spin-nonflip contribution can be written as 


$$
\epsilon_{e x}^{n o n f l i p}=\frac{\alpha_{c}}{4 \pi^{5}}\left(I\left(k_{F}^{+}\right)+I\left(k_{F}^{-}\right)\right)
$$

where $\alpha_{c}$ is the fine structure constant of QCD, $\alpha_{c}=g^{2} / 4 \pi$. The integral $I\left(k_{F}^{i}\right)(i= \pm)$ is given by

$$
I\left(k_{F}^{i}\right)=\frac{\pi^{2}}{2} \xi_{i}\left(\xi_{i}-\frac{4 m_{q}}{3} \kappa_{i}\right)+\tilde{I}\left(k_{F}^{i}\right)
$$

with $\xi_{i}=E_{F}^{i} k_{F}^{i}-m_{q}^{2} \ln \left(E_{F}^{i}+k_{F}^{i} / m_{q}\right)$ and $\kappa_{i}=k_{F}^{i}-m_{q} \ln \left(E_{F}^{i}+k_{F}^{i} / m_{q}\right)$, and the remaining integral

$$
\tilde{I}\left(k_{F}^{i}\right)=\frac{2 \pi^{2} m_{q}}{3}\left[\int_{0}^{k_{F}^{i}} q d q \frac{\left(E_{q}+m_{q}\right)\left(E_{q}+2 m_{q}\right)+m_{q}^{2}}{E_{q}\left(E_{q}+m_{q}\right)} K_{1}\left(q, k_{F}^{i}\right)-\int_{0}^{k_{F}^{i}} \frac{q d q}{E_{q}+m_{q}} K_{2}\left(q, k_{F}^{i}\right)\right] \text {, }
$$

with the functions,

$$
K_{1}\left(q, k_{F}^{i}\right)=\left(E_{F}^{i}-E_{q}\right) \ln \left|\frac{m_{q}^{2}-E_{F}^{i} E_{q}+k_{F}^{i} q}{m_{q}^{2}-E_{F}^{i} E_{q}-k_{F}^{i} q}\right|-2 q \ln \left(\frac{E_{F}^{i}+k_{F}^{i}}{m_{q}}\right)
$$

and

$$
K_{2}\left(q, k_{F}^{i}\right)=\frac{1}{2}\left[\left(k_{F}^{i 2}-q^{2}\right) \ln \left|\frac{m_{q}^{2}-E_{F}^{i} E_{q}+k_{F}^{i} q}{m_{q}^{2}-E_{F}^{i} E_{q}-k_{F}^{i} q}\right|+q E_{q} \ln \left(\frac{E_{F}^{i}-k_{F}^{i}}{E_{F}^{i}+k_{F}^{i}}\right)-2 k_{F}^{i} q\right]
$$

Similarly the spin-flip contribution can be written as

$$
\epsilon_{e x}^{f l i p}=2 \frac{\alpha_{c}}{4 \pi^{5}} J\left(k_{F}^{+}, k_{F}^{-}\right)
$$

The integral $J\left(k_{F}^{+}, k_{F}^{-}\right)$is given by

$$
J\left(k_{F}^{+}, k_{F}^{-}\right)=\frac{\pi^{2}}{2}\left[\xi_{+} \xi_{-}+\frac{2 m_{q}}{3}\left(\xi_{+} \kappa_{-}+\xi_{-} \kappa_{+}\right)\right]+\tilde{J}\left(k_{F}^{+}, k_{F}^{-}\right)
$$

where

$$
\begin{aligned}
\tilde{J}\left(k_{F}^{+}, k_{F}^{-}\right) & =-\frac{\pi^{2} m_{q}}{3}\left[\int_{0}^{k_{F}^{-}} \frac{q d q}{E_{q}+m_{q}}\left(E_{q} K_{1}\left(q, k_{F}^{+}\right)-K_{2}\left(q, k_{F}^{+}\right)\right)\right. \\
& \left.+\int_{0}^{k_{F}^{+}} \frac{p d p}{E_{p}+m_{q}}\left(E_{p} K_{1}\left(p, k_{F}^{-}\right)-K_{2}\left(p, k_{F}^{-}\right)\right)\right] .
\end{aligned}
$$

For the unpolarised $(p=0)$ matter the sum of $I$ and $J$ in Eqs. (13) and (18) gives 


$$
\epsilon_{\text {ex }}^{\text {unol }} \equiv 2 \frac{\alpha_{c}}{4 \pi^{5}}(I+J)=-\frac{\alpha_{c}}{\pi^{3}}\left[k_{F}^{4}-\frac{3}{2}\left\{E_{F} k_{F}-m_{q}^{2} \ln \left(\frac{E_{F}+k_{F}}{m_{q}}\right)\right\}^{2}\right]
$$

with the Fermi energy $E_{F}$ for the unpolarised case, $E_{F}=\left(m_{q}^{2}+k_{F}^{2}\right)^{1 / 2} 12,13$.

In the nonrelativistic case, $k_{F}^{i} \ll m_{q}$, the kinetic energy density is written as

$$
\epsilon_{k i n} \sim \frac{3 k_{F}^{5}}{20 \pi^{2} m_{q}}\left\{(1+p)^{5 / 3}+(1-p)^{5 / 3}\right\} .
$$

The spin-flip contribution becomes tiny in this case, and the dominant contribution for the OGE energy density in Eq.(11) comes from the spin-nonflip contribution, especially $\tilde{I}$ given in Eq. (14), $\tilde{I} \sim-2 \pi^{2} k_{F}^{i 4}$. Thus we find an attractive contribution,

$$
\epsilon_{e x} \sim-\frac{\alpha_{c} k_{F}^{4}}{2 \pi^{3}}\left\{(1+p)^{4 / 3}+(1-p)^{4 / 3}\right\}
$$

The exchange energy contribution is attractive and exactly given by the Coulomb-like (spinnonflip) interaction of gluons. There is no spin-flip contribution in the nonrelativistic limit. The form of the energy densities (21) and (22) is the same as in electron gas. It is the difference of density dependence between these contributions given in Eqs. (21) and (22) which may cause a ferromagnetic instability: as density becomes low enough, all the particles suddenly align their spins and the completely polarised $(p=1)$ matter is energetically favorable. This mechanism is firstly discovered by Bloch for electron gas [8]; in the charge neutral system, electrons tend to avoid the Coulomb repulsion from each other. If all electrons have the same spin state, they can maximally avoid the repulsion by the Pauli principle. On the other hand, the kinetic energy of electrons obviously becomes minimum for the unpolarised $(p=0)$ case. We can see that there is a trade-off between two contributions. Thus the critical density is determined by the mass and the coupling constant.

In the relativistic case there are some different features from the nonrelativistic case. First, there is a spin-flip contribution due to the lower component of the Dirac spinor even for the Coulomb-like interaction. Secondly, the transverse (magnetic) gluons becomes important, where the spin-flip effect is prominent. Finally, the density dependence of kinetic energy as well as the exchange energy is very different [14. Before discussing the general case, we consider the relativistic limit, $k_{F}^{i} \gg m_{q}$; the kinetic energy density behaves like 


$$
\epsilon_{k i n} \sim \frac{3 k_{F}^{4}}{8 \pi^{2}}\left\{(1+p)^{4 / 3}+(1-p)^{4 / 3}\right\}
$$

In this limit, $I\left(k_{F}^{i}\right) \sim \pi^{2} / 2 k_{F}^{i 4}$ and $J\left(k_{F}^{+}, k_{F}^{-}\right) \sim \pi^{2} / 2 k_{F}^{+2} k_{F}^{-2}$. Thus the exchange energy looks like

$$
\epsilon_{e x} \sim \frac{\alpha_{c}}{8 \pi^{3}} k_{F}^{4}\left\{(1+p)^{4 / 3}+(1-p)^{4 / 3}+2\left(1-p^{2}\right)^{2 / 3}\right\}
$$

The kinetic energy is simply an increasing function with respect to the polarisation parameter $p$ and takes a minimum at $p=0$, whereas the exchange energy is the decreasing function and takes a minimum at $p=1$. This is due to the characteristic feature of the spin-flip and the spin-nonflip interactions. Both give a repulsive contribution in the relativistic limit. In the polarised state $(p=1)$ there is no spin-flip interaction and the spin-nonflip contribution remains unchanged in spite of the increase of the Fermi momentum. The difference of the total energy, $\epsilon_{\text {tot }}=\epsilon_{\text {kin }}+\epsilon_{\text {pot }}$, at $p=0$ and $p=1$ reads

$$
\delta \epsilon \equiv \epsilon_{t o t}(p=1)-\epsilon_{t o t}(p=0)=\frac{3 k_{F}^{4}}{8 \pi^{2}}\left(2^{4 / 3}-1\right)\left[1-\frac{4-2^{4 / 3}}{2^{4 / 3}-1} \frac{\alpha_{c}}{\pi}\right] .
$$

Hence if $\alpha_{c}>3.23$ the polarised state is energetically favorable. Thus, ferromagnetism in the relativistic limit has a defferent origin from that in the nonrelativistic case: the spin-flip contribution favors the polarised state.

In Fig. 1 a typical shape of the energy density is depicted as a function of the polarisation parameter $p$, e.g. for the set,$m_{q}=300 \mathrm{MeV}$ of the $s$ quark and $\alpha_{c}=2.2$ as in the MIT bag model [15] ㅇ.

\section{Fig.1}

For small $p \ll 1$, the energy density behaves like

$$
\epsilon_{\text {tot }}-\epsilon_{\text {tot }}(p=0)=\chi^{-1} p^{2}+O\left(p^{4}\right)
$$

\footnotetext{
${ }^{3}$ The difficulties to determine the values of these parameters have been discussed in ref. [6], and we must allow some range for them.
} 
with $\chi^{-1} \equiv \chi_{k i n}^{-1}+\chi_{\text {pot }}^{-1} \cdot \chi$ is proportional to the magnetic susceptibility, and its sign change suggests a ferromagnetic transition. It consists of two contributions: the kinetic energy gives $\chi_{\text {kin }}^{-1}=k_{F}^{5} /\left(3 \pi^{2} E_{F}\right)$ (the Pauli paramagnetism), which changes from $\chi_{\text {kin }}^{-1} \sim O\left(k_{F}^{5}\right)$ at low densities to $\chi_{k i n}^{-1} \sim O\left(k_{F}^{4}\right)$ at high densities. On the other hand, the exchange energy gives $\chi_{\text {pot }}^{-1}=-\frac{2 \alpha_{c} k_{F}^{4}}{9 \pi^{3}}\left[2-\frac{6 k_{F}^{2}}{E_{F}^{2}}+3\left\{E_{F} k_{F}-m_{q}^{2} \ln \left(\frac{E_{F}+k_{F}}{m_{q}}\right)\right\} \frac{k_{F}}{E_{F}^{3}}+2 \frac{k_{F}^{2}}{E_{F}^{2}}\left\{1+\frac{2 m_{q}}{3\left(E_{F}+m_{q}\right)}\right\}\right]$.

In the relativistic limit, $p_{F} \gg m_{q}, \chi_{\text {pot }}^{-1}$ behaves like

$$
\chi_{\text {pot }}^{-1} \sim \frac{\alpha_{c}}{9 \pi^{3}} k_{F}^{4}-\frac{\alpha_{c}}{3 \pi^{3}} k_{F}^{4}=-\frac{2 \alpha_{c}}{9 \pi^{3}} k_{F}^{4}
$$

from Eq. (24), where the first term stems from the spin-nonflip contribution, while the second term from the spin-flip contribution. Then we can see that the effect of the spin-flip contribution overwhelms the one of the spin-nonflip contribution. The potential contribution $\chi_{\text {pot }}^{-1}$ is always negative, and dominant over $\chi_{\text {kin }}^{-1}$ at low densities, while the kinetic contribution $\chi_{k i n}$ is always positive. If $\alpha_{c}>3 \pi / 2=4.7, \chi$ becomes negative over all densities.

For a given set of $m_{q}$ and $\alpha_{c}, \chi$ changes its sign at the critical density, denoted by $n_{c 1}$, and it clearly shows that quark liquid is ferromagnetic below that density. This is equivalent with the sign change of the curvature of the free energy at the origin as a function of the polarisation parameter $p$. Note that the ferromagnetic transition in our case is of the first order, so that it is not sufficient to only see the susceptibility. Even above that density the ferromagnetic phase may be possible. Actually there is a range, $n_{c 1}<n_{q}<n_{c 2}$, which is specified by the condition s.t. $\chi>0$ and $\epsilon<0$. Above the density $n_{c 2}$ there is no longer the stable ferromagnetic phase. However, the metastable state is still possible up to the density $n_{c 3}$, which is specified by the condition s.t. $\eta \equiv \partial \epsilon_{t o t} /\left.\partial p\right|_{p=1}<0$. In Fig.2 we depict the quantities $\chi^{-1}, \delta \epsilon$ and $\eta$ as functions of density, e.g. for the set , $m_{q}=300 \mathrm{MeV}$ and $\alpha_{c}=2.2$. The crossing points with the horizontal axis indicate the critical deisities $n_{c 1}, n_{c 2}$ and $n_{c 3}$, respectively. 
We can see that the ferromagnetic instability occurs at low densities, while the metastable state can exist up to rather high densities.

Finally we show the critical lines satisfying $\chi^{-1}=0, \delta \epsilon=0$ and $\eta=0$ in the QCD parameter $\left(\alpha_{c}\right.$ and $\left.m_{q}\right)$ plane, which seperate the three characteristic regions for a given density. In Fig. 3 they are presented at a density $n_{q}=0.3 \mathrm{fm}^{-3}$. All the lines have the maxima at the intermediate quark mass, and the mechanism of ferromagnetism is different for each side of the maximum, as already discussed. If we take $m_{q}=300 \mathrm{MeV}$ for the $s$ quark or $m_{q} \sim 0 \mathrm{MeV}$ for the $u$ or $d$ quark, and $\alpha_{c}=2.2$ as in the MIT bag model again [15], the quark liquid can be ferromagnetic as a metastable state.

$$
\text { Fig.3 }
$$

In this Letter we have pointed out a possibility of ferromagnetism of quark liquid: quark liquid potentially bears a nature to be ferromagnetic due to the OGE interaction. We have calculated the energy density of the spin polarised quark liquid interacting with the OGE interaction in a relativistic framework, and found that the spin-flip and spin-nonflip contributions exhibit an interesting feature as a function of the polarisation parameter $p$ or density. The ferromagnetic transition in quark liquid is of the first order as in electron gas and there is a metastable state even above the critical density.

We have found that ferromagnetic instability is feasible not only in massive quark system but also in light quark system. The mechanism is different between two systems: the similar mechanism to electron gas can be applied to the former system, while a novel mechanism due to the large spin-flip contribution, which arises as a specific feature of the relativistic system, may be applied to the latter system.

We have seen that the ferromagnetic phase is realized at low densities and the metastable state is plausible up to rather high densities for a reasonable range of the QCD parameters. Our calculation is based on the Hartree-Fock approximation. So we need to examine the higher-order gluon-exchange contributions, especially the ring diagrams, which has been known to be important in the calculation of the susceptibility of electron gas [9,19]. The 
ring diagrams have been calculated for the unpolarised quark matter [12,18], but there is no calculation for the polarised matter.

The lifetime of the metastable state is another remaining problem to be studied. It may be caused by the highly nonperturbative quantum effects. So it should be proportional to the exponential factor at least. Hence it must be difficult to estimate the lifetime reliably.

If a ferromagnetic quark liquid exists stably or metastably around or above nuclear density, it has some implications on the properties of strange quark stars and strange quark nuggets [7]. They should be magnetized in a macroscopic scale. So strange quark nuggets may be no longer a candidate of dark matter in the universe, since they can emit the electromagnetic waves like pulsars.

For a consideration of a possibility to attribute magnetars to strange quark stars in a ferromagnetic phase, we roughly estimate the strength of the magnetic field at the surface of a strange quark star. The stellar parameters of strange quark stars are similar to those for normal neutron stars for the typical mass around $M_{G}=1.4 M_{\odot}$ [16, [17]. The magnetic dipole moment $M_{q}$ amounts to $M_{q}=\mu_{q} \cdot\left(4 \pi / 3 \cdot r_{q}^{3}\right) n_{q}$ for the quark sphere with the radius $r_{q}$, where $\mu_{q}$ is the magnetic moment of each quark, $\mu_{q} \sim \mu_{N}\left(\mu_{N}\right.$ :nuclear magneton $5 \times 10^{-24} \mathrm{erg} \cdot$ gauss $^{-1}$ ) for massive quarks and $10^{2} \mu_{N}$ for light quarks. Then the dipolar magnetic field at the star surface $r=R \simeq 10 \mathrm{Km}$ takes a maximal strength at the poles,

$$
B_{\max }=\frac{8 \pi}{3}\left(\frac{r_{q}}{R}\right)^{3} \mu_{q} n_{q}
$$

which is order of $O\left(10^{15-17}\right) \mathrm{G}$ for $n_{q}=O(0.1) \mathrm{fm}^{-3}$, enough for the magnitude required for magnetars.

One may wonder whether such a strong magnetic field modifies the Fermi sea of quark liquid itself. We can see, however, that the mean magnetic field working on one quark , which is produced by other quarks, vanishes due to the uniform distribution of quarks. Hence the self-consistency is retained even in such a case.

The author thanks M. Matsuzaki and T. Muto for useful discussions and suggestions 
on the manuscript. He also thanks N. Iwamoto for stimulating discussions. This work was supported in part by the Japanese Grant-in-Aid for Scientific Research Fund of the Ministry of Education, Science, Sports and Culture (11640272). 


\section{REFERENCES}

[1] For a review, G. Chanmugam, Annu. Rev. Astron. Astrophys. 30 (1992) 143.

[2] B. Paczyński, Acta. Astron. 41 (1992) 145.

R.C. Duncan and C. Thompson, Astrophys. J. 392 (1992) L19; C. Thompson and R.C.

Duncan, Mon. Not. R. Astron. Soc. 275 (1995) 255.

[3] C. Kouveliotou et al., Nature 393 (1998) 235.

K. Hurley et al., Astrophys. J. 510 (1999) L111.

[4] S.A. Chin and A.K. Kerman, Phys. Rev. Lett. 43 (1979) 1292.

[5] E. Witten, Phys. Rev. D30 (1984) 272.

[6] E. Farhi and R.L. Jaffe, Phys. Rev. D30 (1984) 2379.

[7] For a review, J. Madsen, astro-ph/9809032

[8] F. Bloch, Z. Phys. 57 (1929) 545.

[9] D.M. Ceperley and B.J. Adler, Phys. Rev. Lett. 45 (1980) 566.

[10] V.B. Berestetsii, E.M. Lifshitz and L.P. Pitaevsii, Relativistic Quantum Theory(Pergamon Press, 1971).

[11] e.g.,C. Itzykson and J.-B. Zuber, Quantum Field Theory(McGraw-Hill Inc., 1980).

[12] G. Baym and S.A. Chin, Nucl. Phys. A262 (1976) 527.

[13] I.A. Akhiezer and S.V. Peletminskii, JETP 11 (1960) 1316.

[14] e.g.,R. Tamagaki and T. Tatsumi, Prog. Theor. Phys. Suppl. 112 (1993) 277.

[15] T. DeGrand et al., Phys. Rev. D12 (1975)2060.

[16] P. Haensel, J.L. Zdunik and R. Schaeffer, Astron. Astrophys. 160 (1986) 121.

[17] C. Alcock, E. Farhi and A. Olinto, Astrophys. J. 310 (1986) 261. 
[18] J.I. Kapusta, Finite-Temperature field theory(Cambridge Univ. Press, 1989).

[19] K.A. Brueckner and K. Sawada, Phys. Rev. 112 (1957) 328. 


\section{FIGURES}

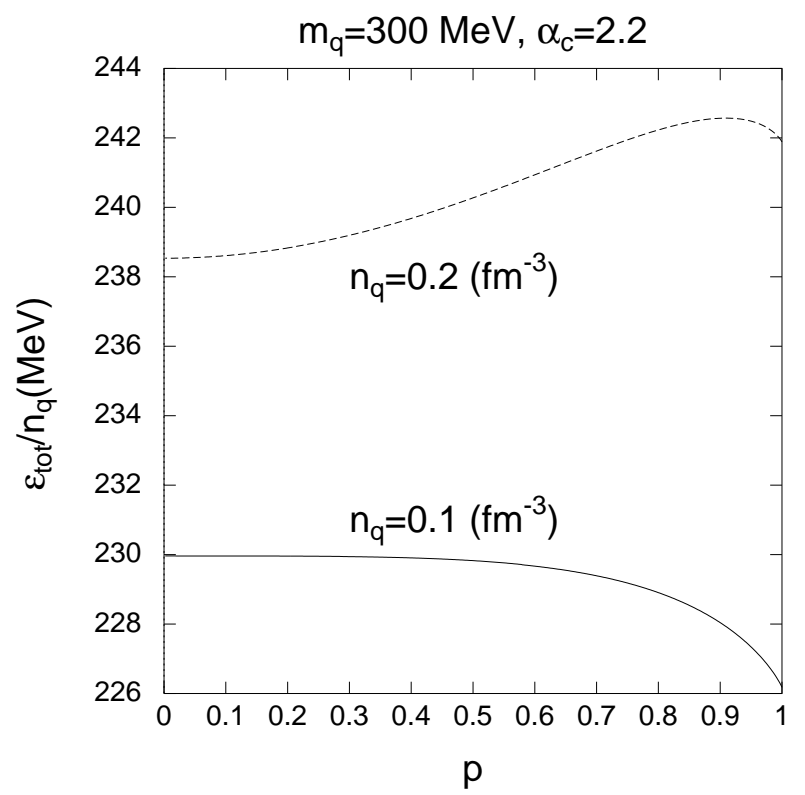

FIG. 1. Plot of the energy density as a function of the polarisation parameter at $n_{q}=0.1 \mathrm{fm}^{-3}$ and $n_{q}=0.2 \mathrm{fm}^{-3}$.

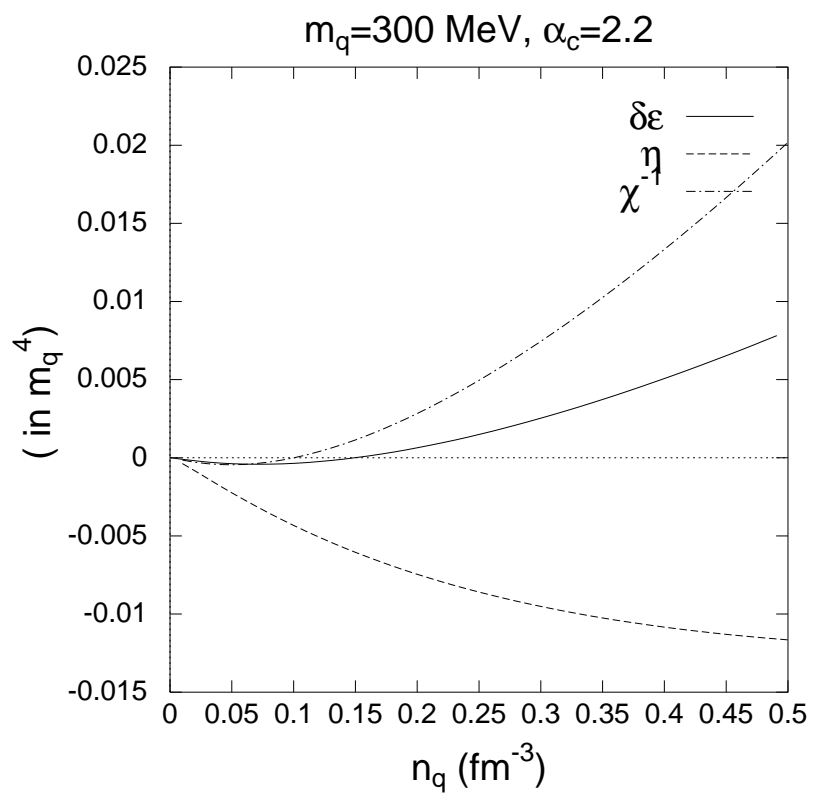

FIG. 2. Density dependence of $\chi^{-1}, \delta \epsilon$ and $\eta$. In this case, $n_{c 1} \simeq 0.1 \mathrm{fm}^{-3}, n_{c 2} \simeq 0.15 \mathrm{fm}^{-3}$ and $n_{c 3}>0.5 \mathrm{fm}^{-3}$. 


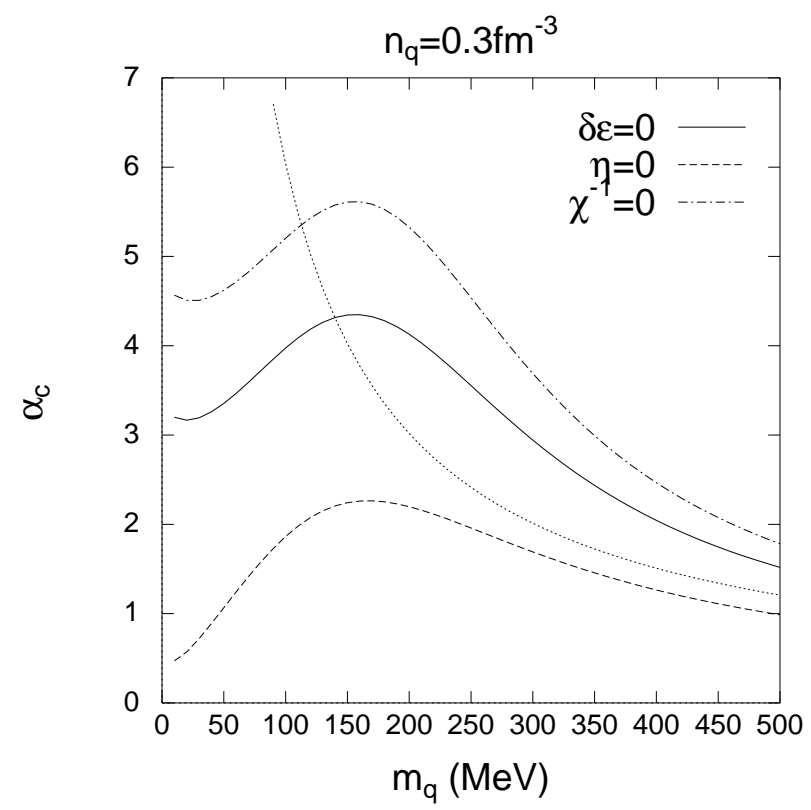

FIG. 3. Phase diagram in the QCD parameter plane. The region above the solid curve is in a ferromagnetic phase. The region bounded by the solid and dased curves is in a paramagnetic phase, but a metastable ferromagnetic state is possible. The dash-dotted curve shows the boundary where the susceptibility is vanished. The dotted curve shows the corresponding one to the solid curve with the nonrelativistic formulae given in Eqs. (21) and (22) for comparison. 